\title{
EXTRACHROMOSOMAL ELEMENTS IN A SUPER-SUPPRESSION SYSTEM OF YEAST
}

\section{A NUCLEAR GENE CONTROLLING THE INHERITANCE OF THE EXTRACHROMOSOMAL ELEMENTS}

\author{
C. S. H. YOUNG and B. S. COX \\ Botany School, South Parks Road, Oxford OX1 3RA
}

Received 24.vii.70

\section{InTRODUGTION}

SUPER-SUPPRESSION in the yeast Saccharomyces cerevisiae was first reported by Hawthorne and Mortimer (1963). It may be defined as a state in which the phenotypes of specific alleles at a variety of loci are simultaneously reversed to a pseudo-wild type condition by the presence of a mutation at an external locus. It is clear from evidence on the mutability of suppressor loci and of suppressible alleles (Hawthorne, 1969a,b) the polypeptide fragments produced by suppressible alleles (Manney, 1969) and the amino acid changes in proteins produced by such alleles when they are reverted or suppressed (Gilmore, Stewart and Sherman, 1968), that super-suppression is closely akin to the more fully understood "nonsense" suppression of procaryotes (reviews: Gorini and Beckwith, 1966; Garen, 1968). Thus super-suppression probably results from an alteration to a minor species of transfer RNA such that it is able to translate the "stop " codons UAA, UAG or UGA occurring internally in the messenger RNA transcribed from suppressible alleles.

In 1965, Cox discovered that when certain non-suppressed revertants from a suppressed strain of yeast were back-crossed, the revertant phenotype failed to reappear in the haploid progeny. Further crosses supported the hypothesis that the action of the super-suppressor $\left(S U Q_{5}\right)$ was dependent upon the presence of an extrachromosomal element, $\psi$. It was proposed that those strains capable and incapable of suppression should be given the genotypes $S U Q_{5} \psi^{+}$and $S U Q_{5} \psi^{-}$respectively. The failure of the nonsuppressed phenotype to reappear in haploid offspring following a cross between $S U Q_{5} \psi^{-}$and $S U Q_{5} \psi^{+}$strains could be explained if $\psi^{+}$elements were distributed to all the products of meiosis. [This situation has a parallel in the behaviour of "neutral petite " $\times$ wild type crosses in which the respiratory defective phenotype fails to reappear in any of the haploid offspring (Ephrussi, Hottinguer and Tavlitski, 1949).] These results suggest the involvement, directly or indirectly, of extrachromosomal genetic elements in protein synthesis.

In an attempt to isolate further mutants which were non-suppressed because of mutation in the extrachromosomal element(s), another class of mutant was observed in which the hereditary continuity of $\psi$ appeared to be affected by mutation in a nuclear gene. This paper describes the characteristics of one of these mutants. 


\section{Materials and Methods}

(i) Strains

The strains of Saccharomyces cerevisiae used in this investigation all derive from our laboratory. They are:

$$
\begin{aligned}
& 163 / 9 c \text { white : } \alpha \text {, ade } 2-1, S_{Q_{5}}, \psi^{+} \\
& 170 / 2 c \text { white : a, ade } 2-1, S_{Q_{5}}, \psi^{+} \\
& 170 / 2 c \text { red }: \text { a, ade } 2-1, S_{Q_{5}}, \psi^{-} \\
& \text {1c : } \alpha \text {, ade2-1, met- } t^{-} \text {tyr }{ }^{-}, S_{Q_{5}}, \psi^{+} \quad \text { white, prototrophic } \\
& \} \text { white, prototrophic } \\
& \text { red, adenine requiring }
\end{aligned}
$$

ade2-1 is a mutation conferring a requirement for adenine. It is the allele $a d_{2 \cdot 1}$ described by Hawthorne and Mortimer (1963), and is said to be an ochre mutation (Hawthorne, 1969b). It is important to note that ade2-1 when unsuppressed, confers a red colour on the strain. Otherwise, yeast colonies are white. met and tyr are mutations conferring requirements for methionine and tyrosine respectively. 1965).

All three alleles are suppressible by the suppressor $S_{Q_{5}}\left(S U Q_{5}\right.$ in Cox,

$\psi^{+}$and $\psi^{-}$are the extrachromosomal alleles which respectively allow or prevent the functioning of $S_{Q_{5}}$.

$a$ and $\alpha$ are mating-type alleles.

\section{(ii) Media}

YC : A complete medium described by Cox and Bevan (1962), solidified with Oxoid agar No. 3 to 2 per cent. w/v.

YNB : A basal medium prepared from Yeast Nitrogen Base without amino acids (Difco) 0.67 per cent. $w / v$ and 1 per cent. w/v glucose, solidified with Oxoid Ionagar No. 2 to 1.25 per cent w/v. It is supplemented, where necessary, with amino acids and bases to final concentrations of 20 and $10 \mu \mathrm{g} \cdot \mathrm{ml}^{-1}$ respectively.

SM : An acetate sporulation medium based on that described by Pomper, Daniels and McKee (1954), solidified with Oxoid Ionagar No. 2 to 1.5 per cent. $\mathrm{w} / \mathrm{v}$.

\section{(iii) Growth conditions}

Inocula on $\mathrm{YC}$ and $\mathrm{YNB}$ plates were grown at $28^{\circ} \mathrm{C}$. For sporulation, inocula from three-day-old diploid colonies were streaked on SM and incubated for four days at $25^{\circ} \mathrm{C}$. Asci were dissected on the fourth day.

\section{(iv) Micromanipulation}

Individual zygotes were isolated from mass mating mixtures on YC plates, and asci were dissected for tetrad analysis, using a Singer micromanipulator (Barer and Saunders-Singer, 1948). Ascus walls were digested using the snail-crop extract techniques of Johnston and Mortimer (1959).

\section{(v) Replica plating}

Colonies were replicated using Whatman No. $1,11 \mathrm{~cm}$. diameter filter paper as the replicating surface. Replica plates were examined after three, four and five days. 


\section{Results \\ (i) Isolation of mutant $\mathrm{U}_{16}$}

Strain $1 c$ is white and prototrophic but mutations occurring in either $S_{Q_{5}}$ or in $\psi$ may cause the failure of suppression which will result in a triple requirement for adenine, methionine and tyrosine. Cells of strain $1 c$ were irradiated with ultraviolet light and then plated on YG. After

\section{TABLE 1}

Replication of red isolates, following UV irradiation of strain lc

\begin{tabular}{|c|c|}
\hline Phenotypes & Nos. obtained \\
\hline $\begin{array}{l}\text { ade }^{-} \text {met }^{-} \text {tyr }^{-} \\
\text {ade }^{-} \text {MET }^{+} \text {TYR } \\
\text { ade }^{-} \text {met }^{-} \text {TYR }\end{array}$ & $\begin{array}{c}18 \\
5 \\
1\end{array}$ \\
\hline $\begin{array}{l}\text { Total no. of colonies examined } \\
\text { (white and red) }\end{array}$ & 8359 \\
\hline Percentage survival, cf. control & $5 \cdot 8$ \\
\hline
\end{tabular}

four days, red colonies were selected and tested by replication on YNB plates suitably supplemented (table 1). The 18 polyauxotrophs obtained were then crossed to a $\psi^{-}$strain, $170 / 2 c$ red, and to a $\psi^{+}$strain, $170 / 2 c$ white. The expected results from such crosses are:

(a) If mutation has occurred in $\psi$, then the cross to $170 / 2 \mathrm{c}$ red should yield only red offspring since neither parent contributes a functional $\psi^{+}$

TABLE 2

Crosses of $\mathrm{U}_{16}$ to $170 / 2 \mathrm{c}$ red and $170 / 2 \mathrm{c}$ white

\begin{tabular}{|c|c|c|c|c|c|}
\hline \multirow[b]{2}{*}{ Cross } & \multicolumn{2}{|c|}{ Diploid phenotype } & \multicolumn{3}{|c|}{$\begin{array}{l}\text { Segregation in tetrads of growth require- } \\
\text { ments }(+:-) \text { and of mating type } a: a\end{array}$} \\
\hline & Colour & Requirement & Adenine & Methionine & Mating type \\
\hline " red" $\times U_{16}$ & Red & Adenine & $11 \times 0: 4$ & $\begin{array}{r}10 \times 2: 2 \\
1 \times 4: 0\end{array}$ & \} Not tested \\
\hline \multirow[t]{3}{*}{ "white" $\times U_{16}$} & Pink & Adenine & $4 \times 0: 4$ & $4 \times 2: 2$ & \multirow{3}{*}{$8 \times 2: 2$} \\
\hline & & & $2 \times 1: 3$ & $\begin{array}{l}1 \times 3: 1 \\
1 \times 2: 2\end{array}$ & \\
\hline & & & $\begin{array}{l}2 \times 2: 2 \\
-\end{array}$ & $\begin{array}{l}1 \times 3: 1 \\
1 \times 2: 2\end{array}$ & \\
\hline
\end{tabular}

Notes: 1 . The tyrosine marker in $U_{16}$ had reverted at the time the crosses were performed.

2. The crosses are heterozygous for met. In the $3: 1$ segregations in the lower cross, one of the MET+ clones was also white $\mathrm{AD}^{+}$. The 4:0 segregation in the upper cross is anomalous.

particle. On the other hand, in the cross to $170 / 2 c$ white the mutant phenotypes should rarely, if ever, reappear.

(b) If mutation has occurred in $S_{Q_{5}}$, then both crosses should yield segregations of 2 red : 2 white in tetrad analysis, since the diploids are $\psi^{+}$ and heterozygous for $S_{Q_{5}}$.

Of the 18 mutants tested, three gave pattern (a), one gave pattern (b) and the rest gave a variety of patterns of which that from $U_{16}$ is an example (table 2). In the cross to $170 / 2 \mathrm{c}$ red, neither the diploid nor any of the 
haploid offspring were suppressed. This means that $U_{16}$ is $\psi$, since any $\psi^{+}$particles brought into the cross by the mutant would allow the $S_{Q_{5}}$ allele, known to be present in $170 / 2 \mathrm{c}$ red, to act as a suppressor both in the diploid and in two out of four spore-clones per tetrad. However in the cross to $170 / 2 c$ white, segregation of the mutant phenotypes in the tetrads is irregular and the mutant rather than the wild-type phenotype appears in the majority of the haploid offspring. This is the reverse of the pattern

a. Cytoplasm containing "suppressive", non-functioning $\psi$ particles

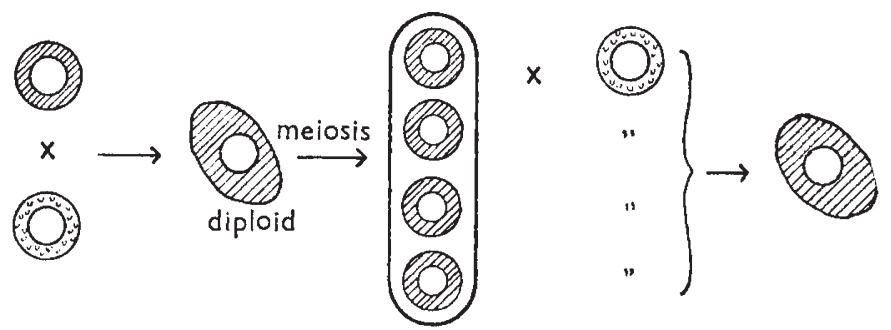

All diploids segregate a majority of red haploid offspring after meiosis

b. Nuclear allele causing elimination or mutation of $\psi$ particles

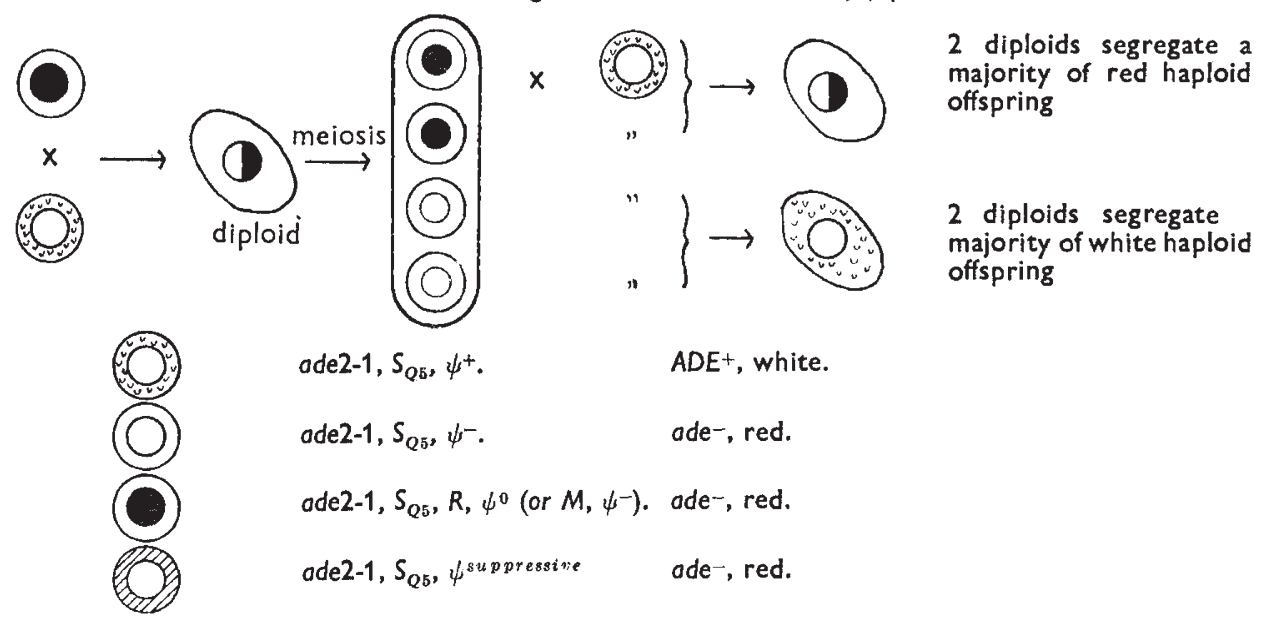

FIG. 1.-Alternative explanations of the behaviour of the non-suppressed revertant $U_{16}$, showing the consequences of crossing it with a suppressed white strain (ade2-l, $\left.S_{Q 5} \psi^{+}\right)$.

expected if the cross were between $\psi^{-}$and $\psi^{+}$strains (pattern $(a)$ ). Two general explanations may be advanced for this behaviour:

(a) $U_{16}$ possesses a "suppressive" non-functional $\psi$ particle, perhaps analogous to the suppressive factor in certain respiratory-deficient yeast strains (Ephrussi, Margerie-Hottinguer and Roman, 1955). During proliferation of the diploid from the $U_{16} \times 170 / 2 c$ white zygote, the suppressive particles in some way exclude the funcional $\psi^{+}$particles, so that few are distributed to haploid offspring at meiosis with a consequent reduction in the number of suppressed spore-clones.

(b) $U_{16}$ contains a nuclear allele which prevents the expression of $\psi^{+}$ particles. It also slows down or prevents the replication of $\psi^{+}$particles or causes them to mutate to a non-functional condition. The allele is 
dominant in the heterozygous diploid formed from the cross between $U_{16}$ and $170 / 2 c$ white; as in explanation $(a)$, the result will be a lowering of the number of $\psi^{+}$particles distributable at meiosis.

\section{(ii) Crosses to determine the genetic basis of the behaviour of $\mathrm{U}_{16}$}

The two explanations may be tested in the following way (fig. 1). Four red non-suppressed spore-clones from a tetrad from the $\operatorname{cross} U_{16} \times 170 / 2 \mathrm{c}$ white may be back-crossed to white suppressed strains of appropriate mating type. If explanation $(a)$ is correct, all four crosses should yield similar results, namely that the majority of haploid offspring from each cross

TABLE 3

Croses between four red spore-clones, a-d, from a tetrad from the $\mathrm{U}_{16} \times 170 / 2 \mathrm{c}$ white cross and appropriate white (ade2-1, $\mathrm{S}_{Q 5} \psi^{+}$) strains

\begin{tabular}{|c|c|c|}
\hline Cross & $\begin{array}{c}\text { Tetrad analysis } \\
\text { (white:red) }\end{array}$ & $\begin{array}{l}\text { Spore-clone totals } \\
\text { (white:red) }\end{array}$ \\
\hline$a \times 170 / 2 c$ white & $\begin{array}{l}6 \times 4: 0 \\
1 \times 3: 1 \\
1 \times 2: 2 \\
1 \times 3: 0\end{array}$ & $32: 3$ \\
\hline$b \times 163 / 9 c$ white & $\begin{array}{r}1 \times 1: 3 \\
13 \times 0: 4\end{array}$ & $1: 55$ \\
\hline$c \times 170 / 2 c$ white & $\begin{array}{l}6 \times 2: 2 \\
2 \times 1: 3 \\
4 \times 0: 4 \\
1 \times 1: 1 \\
1 \times 0: 1\end{array}$ & $15: 36$ \\
\hline$d \times 163 / 9 c$ white & $\begin{array}{r}10 \times 4: 0 \\
1 \times 3: 0 \\
1 \times 1: 1 \\
1 \times 0: 1\end{array}$ & $45: 2$ \\
\hline
\end{tabular}

should be red and non-suppressed. This is because the suppressive, nonfunctional $\psi$ particle(s) will be brought into each cross by the respective spore-clone parents. Explanation $(b)$, on the other hand, predicts that only two of the four clones will contain the nuclear gene responsible for loss of suppressor function and hence only two crosses will yield a majority of red offspring. The other two clones will be normal $\psi^{-}$strains and on crossing to $\psi^{+}$strains should yield a majority of white offspring. Table 3 sets out the results obtained from such crosses and it would appear that explanation $(b)$ is supported. Only crosses $b \times 163 / 9 c$ white and $c \times 170 / 2 c$ white yield a majority of red offspring and the irregular pattern of segregation in the tetrads mimics that of the parental cross $U_{16} \times 170 / 2 \mathrm{c}$ white (table 2). Spore clones $a$ and $d$ behave as $\psi^{-}$strains.

Not all tetrads from the cross $U_{16} \times 170 / 2 c$ white segregated 4 red :0 white spore clones. This could be explained by assuming that the nuclear allele in $U_{16}$ was incapable of eliminating all $\psi^{+}$particles before meiosis, so that some were distributed to spores which contained the wild-type nuclear allele. These spores would give rise to suppressed clones. (Explanation $(b)$ predicts that never more than two clones per tetrad should be suppressed, as is the case in the results obtained (table 2).) To test if the 
white clone in a 3 red:1 white tetrad from the cross $U_{16} \times 170 / 2 c$ white was $\psi^{+}$, a set of intratetrad crosses was performed (table 4). Cross $a \times d$ yields only white haploid offspring. This is a typical result for a $\psi^{+} \times \psi^{-}$cross. Cross $a \times c$, on the other hand, behaves similarly to $U_{16} \times 170 / 2 c$ white in that phenotype segregation is irregular and a majority of haploid offspring

TABLE 4

Intra-tetrad crosses within an $F_{1}$ tetrad from the cross $\mathrm{U}_{16} \times 170 / 2 \mathrm{c}$ white in which three spore-clones are red and one white

\begin{tabular}{|c|c|c|}
\hline Cross & $\begin{array}{l}\text { Tetrad analysis } \\
\text { (white: red) }\end{array}$ & $\begin{array}{l}\text { Spore-clone totals } \\
\text { (white:red) }\end{array}$ \\
\hline$a$ (white) $\times c$ (red) & $\begin{array}{l}2 \times 2: 2 \\
2 \times 1: 3 \\
2 \times 0: 4 \\
1 \times 2: 1 \\
3 \times 1: 2 \\
1 \times 0: 3 \\
1 \times 1: 1 \\
1 \times 0: 1\end{array}$ & $12: 30$ \\
\hline$a($ white $) \times d($ red $)$ & $\begin{array}{r}16 \times 4: 0 \\
1 \times 3: 0 \\
1 \times 1: 0\end{array}$ & $68: 0$ \\
\hline$b(\mathrm{red}) \times c(\mathrm{red})$ & $\begin{array}{r}15 \times 0: 4 \\
1 \times 0: 2 \\
1 \times 0: 1\end{array}$ & $0: 63$ \\
\hline$b(\mathrm{red}) \times d(\mathrm{red})$ & $\begin{array}{r}17 \times 0: 4 \\
3 \times 0: 3\end{array}$ & $0: 77$ \\
\hline
\end{tabular}

are red. From these two crosses it is possible to deduce that spores $a$ and $d$ contained the wild-type allele of the mutant gene present in $U_{16}$ and that spore $a$ received functional $\psi^{+}$particle(s) as well. If the mutant allele is designated $R$, the wild-type being $r$, then the genotypes of these segregants would be:

$$
\begin{aligned}
& a=\operatorname{ade} 2-1 S_{Q_{5}} r \psi^{+} \text {(white) } \\
& b=\operatorname{ade} 2-1 S_{Q_{5}} R \quad \text { (red) } \\
& c=\operatorname{ade2-1} S_{Q_{5}} R \quad \text { (red) } \\
& d=\operatorname{ade2-1} S_{Q_{5}} r \psi^{-} \text {(red) }
\end{aligned}
$$

the $\psi$-status of $b$ and $c$ being uncertain.

\section{(iii) Progressive effect of the nuclear allele}

The action of the nuclear allele in diploids would seem to be incomplete in that it does not eliminate all $\psi^{+}$particles before meiosis. It was decided to test whether or not the extent of elimination altered with time. Accordingly, a mass mating was set up on a YG plate and eight zygotes were isolated and allowed to proliferate. At the same time an inoculum from the mating mixture was transferred to an SM plate to induce sporulation in the zygotes directly. Two of the isolated zygotes were sampled by placing inocula on SM plates after two days' growth on YG, three were sampled after three days and the remaining three after four days. The data show 
TABLE 5

Sporulation of diploid clones of different ages from the cross $\mathrm{U}_{16} \times 170 / 2 \mathrm{c}$ white

\begin{tabular}{|c|c|c|c|c|c|c|c|c|c|c|}
\hline \multirow{2}{*}{$\begin{array}{c}\text { Days of growth } \\
\text { before } \\
\text { sporulation }\end{array}$} & \multirow{2}{*}{\multicolumn{2}{|c|}{$\begin{array}{l}\text { No. of diploid } \\
\text { clones analysed }\end{array}$}} & \multicolumn{6}{|c|}{ Tetrad analysis (white:red) } & \multirow{2}{*}{$\begin{array}{l}\text { Spore-clone } \\
\text { totals* }\end{array}$} & \multirow{2}{*}{$\begin{array}{l}\text { Percentage } \\
\text { red spore } \\
\text { clones }\end{array}$} \\
\hline & & & $2: 2$ & $1: 3$ & $0: 4$ & $2: 1$ & $1: 2$ & $0: 3$ & & \\
\hline 0 & $21 z$ & rotes & 17 & 1 & - & - & 1 & - & $36: 39$ & 52 \\
\hline \multirow[t]{3}{*}{2} & 2 & No. 1 & 10 & 3 & 1 & - & 1 & 1 & $24: 38$ & 61 \\
\hline & & 2 & 13 & 3 & 1 & - & 1 & $\ldots$ & $30: 42$ & 58 \\
\hline & & $1+2$ & 23 & 6 & 2 & - & 2 & 1 & $54: 80$ & 60 \\
\hline \multirow[t]{4}{*}{3} & 3 & No. 1 & 5 & 2 & 6 & 1 & 1 & 2 & $15: 51$ & 77 \\
\hline & & 2 & 9 & 6 & 2 & 1 & - & 2 & $26: 51$ & 66 \\
\hline & & 3 & 7 & 7 & 4 & - & 1 & - & $22: 53$ & 71 \\
\hline & & $1+2+3$ & 21 & 15 & 12 & 2 & 2 & 4 & $63: 155$ & 71 \\
\hline \multirow[t]{4}{*}{4} & 3 & No. 1 & 5 & 4 & 6 & - & 1 & - & $15: 52$ & 78 \\
\hline & & 2 & 10 & 5 & 3 & - & - & 1 & $25: 50$ & 67 \\
\hline & & 3 & 4 & 4 & 5 & - & - & 3 & $12: 49$ & 80 \\
\hline & & $1+2+3$ & 19 & 13 & 14 & - & 1 & 4 & $52: 151$ & 74 \\
\hline
\end{tabular}

* Includes observations on tetrads with fewer than four germinating ascospores.



FIg. 2.-The progressive effect of the nuclear allele in eliminating $\psi^{+}$particles from diploids heterozygous for it. 
that the zygotes which were immediately sporulated yielded a precise $2: 2$ segregation of adenine requirement in the tetrads. Those which were allowed to grow on yielded, with time, an increasing proportion of tetrads with an excess of non-suppressed cultures. This suggests a progressive elimination (or mutation) of $\psi^{+}$particles in the growing diploids (table 5 and fig. 2).

\section{(iv) Sectoring}

Normally, crosses between $\psi^{+}$and $\psi^{-}$strains give diploid and haploid offspring which are phenotypically pure and do not sector. However, in crosses between $U_{16}$ and $170 / 2 c$ white the haploid segregants often exhibited this phenomenon. Only two clones per tetrad were white or sectored red and white, the other two clones being red. This suggested that sectoring only occurred in the absence of the nuclear allele responsible for loss of suppressor function and that white sectors were $\psi^{+}$. To test this, two white sectors were isolated and purified and crossed to $170 / 2 \mathrm{cred}$. The diploids were white, suppressed and on sporulation gave haploid segregations of 53:2 and 26:2 white:red. This confirms that the sectors were $\psi^{+}$and that the nuclear allele responsible for loss of suppressor function was absent.

\section{(v) Effects of the nuclear allele on other extrachromosomal determinants}

$U_{16}$ is not petite. This suggests that the nuclear allele's effect is confined to $\psi$ and does not affect $\rho$, the extrachromosomal determinant for respiratory competency. Preliminary results also indicate that the allele has no effect on an extrachromosomally-inherited determinant for erythromycin resistance.

\section{Discussion}

Extrachromosomal elements whose replication is known to be controlled by nuclear genes include the killer characters in Paramecium (Sonneborn, 1943) and yeast (Somers and Bevan, 1969), the "red-variant" determinant in Aspergillus nidulans (Grindle, 1964) and the $\rho$ determinant in yeast (Sherman, 1963). An example of a nuclear gene which causes mutation in extrachromosomal determinants is "iojap" in maize and its effect on plastid genes (Rhoades, 1946). It is not possible to conclude whether the situation described in the present paper results from elimination or mutation of $\psi^{+}$particles, but the nature of the control by the nuclear allele is intriguing; for example, the allele is dominant, its action is slow but progressive and it appears to have no effect on other extrachromosomal determinants.

Elimination of $\psi^{+}$particles in $U_{16}$ could be explained by assuming that a nuclear gene, necessary for their replication, had mutated to an inactive state. But the mutated nuclear gene would not be expected to be dominant in the $U_{16} \times 170 / 2 c$ white cross, since the wild-type allele would presumably allow $\psi^{+}$replication. One model to account for the dominance of the mutant allele would be that the nuclear gene provides a repressor of $\psi$ replication. Normally, during cell growth and division the repressor product is inactivated by an apo-repressor so that $\psi$ replication proceeds in step with nuclear replication. In the mutant, the repressor product is 
insensitive to inactivation so that $\psi$ replication is continuously repressed. In diploids heterozygous for the controlling gene, the mutant repressor product would still be present. It should be noted that the action of the nuclear allele is immediate with respect to the expression of $\psi^{+}$particles because diploid clones from the cross $U_{16} \times 170 / 2 c$ white are homogeneously red and adenine requiring despite the fact that many $\psi^{+}$particles are still present and may be distributed to spores at meiosis.

An alternative model is that the nuclear gene causes mutation in $\psi^{+}$ particles. But this part of its activity could not be immediate since nonmutant $\psi^{+}$particles are recovered later. The model based on a repression of replication would, however, allow for a "segregational lag " even if this repression, as well as the repression of function, were immediate.

The specificity of the mutant nuclear allele, $R$, for $\psi$ may reveal complexities within the yeast extrachromosomal genome. If $\psi$ is linked to $\rho$ and the determinant for erythromycin resistance, then the specificity of action implies that elimination or mutation can occur at specific points on an "extrachromosomal chromosome". Alternatively, $\psi$ and the other two determinants may not be linked, in which case the specificity lies in an ability to recognise different hereditary particles in the cytoplasm. Evidence for the separateness of $\psi$ and $\rho$ and the determinant for erythromycin resistance will be presented in another communication.

\section{SUMmary}

1. In a super-suppression system of yeast, the nuclear gene $S_{Q_{5}}$ acts as a suppressor in conjunction with an extrachromosomal element designated $\psi^{+}$.

2. This paper demonstrates that a non-suppressed isolate from an $S_{Q_{5}} \psi^{+}$strain contains a nuclear allele which eliminates or mutates $\psi^{+}$ particles.

3. The allele is dominant, and has been designated $R$.

4. It prevents the expression of $\psi^{+}$particles in diploids and haploids and causes their progressive elimination during the proliferation of zygotes.

5. Other results indicate it has no effect on the extrachromosomal determinants for respiratory competence or erythromycin resistance.

Acknowledgment.-This work was done while one author (C. S. H. Y.) held a Medical Research Council Research Studentship.

\section{REFERENCES}

BARER, R., AND SAUNDERS-SINGER, A. E. 1948. A new single-control micromanipulator. Q.7. microsc. Sci., 89, 439-447.

cox, B. s. 1965. $\Psi$, a cytoplasmic suppressor of super-suppressor in yeast. Heredity, 20, 505-521.

cox, B. S., ANd beVan, E. A. 1962. Aneuploidy in yeast. New Phytol., 61, 342-355.

EPHRUSSI, B., hOtTINGUER, H., AND tAvlitski, H. 1949. Action de l'acriflavine sur les levures. II. Etude génétique du mutant "petite colonie". Annales Inst. Pasteur, Paris, 77, 64-83.

EPHRUSSI, B., MARGERIE-HOTTINGUER, H., AND ROMAN, H. 1955. Suppressiveness: a new factor in the genetic determinism of the synthesis of respiratory enzymes in yeast. Proc. natn. Acad. Sci. U.S.A., 41, 1065-1071. 
Garen, A. 1968. Sense and nonsense in the genetic code. Science, N.Y., 160, 149-159.

Gilmore, R. A., stewart, J. W., AND Sherman, F. 1968. Amino acid replacements resulting from super-suppression of a nonsense mutant of yeast. Biochim. biophys. Acta, 161, 270-272.

GRINDLE, M. 1964. Nucleocytoplasmic interactions in the " red" cytoplasmic variant of Aspergillus nidulans. Heredity, 19, 75-95.

gorini, l., AND beckwith, J. R. 1966. Suppression. Ann. Rev. Microbiol., 20, 401-422.

HAWTHORNE, D. C. 1969a. The selection of nonsense suppressors in yeast. Mutation Res., 7, 187-197.

HAWTHORNE, D. C. 1969b. Identification of nonsense codons in yeast. 7. molec. Biol., 43, 71-75.

hAWTHORNE, D. C., AND MORTIMER, R. K. 1963. Super-suppressors in yeast. Genetics, Princeton, 48, 617-620.

Johnston, J. R., AND MORTIMER, R. K. 1959. Use of snail digestive juice in isolation of yeast spore tetrads. F. Bact., 78, 292.

MANNEY, T. R. 1969. Evidence for chain termination by super-suppressible mutants in yeast. Genetics, Princeton, 60, 719-753.

POMPER, S., DANiels, K. M., AND MCKEe, D. w. 1954. Genetic analysis of polyploid yeast. Genetics, Princeton, 39, 343-355.

Rhoades, м. м. 1946. Plastid Mutations. Cold Spring Harb. Symp. Quant. Biol., 11, 202-207. sherman, F. 1963. Respiration-deficient mutants of yeast. I. Genetics. Genetics, Princeton, 48, 375-385.

SOMERS, J. M., AND BEVAN, E. A. 1969. The inheritance of the killer character in yeast. Genet. Res., 13, 71-83.

SONNEBORN, T. м. 1943. Gene and cytoplasm. I. The determination and inheritance of the killer character in variety 4 of Paramecium aurelia. Proc. natn Acad. Sci. U.S.A., 29, 329-338. 